\title{
Questes
}

\section{Conclusion : Se prendre au jeu}

\section{Amandine Mussou et Laëtitia Tabard}

\section{(2) OpenEdition}

\section{Journals}

Édition électronique

URL : https://journals.openedition.org/questes/673

DOI : 10.4000/questes.673

ISSN : 2109-9472

\section{Éditeur}

Les Amis de Questes

\section{Édition imprimée}

Date de publication : 15 février 2010

Pagination : 103-106

ISSN : 2102-7188

\section{Référence électronique}

Amandine Mussou et Laëtitia Tabard, «Conclusion : Se prendre au jeu », Questes [En ligne], 18| 2010, mis en ligne le 01 janvier 2014, consulté le 28 juin 2022. URL : http://journals.openedition.org/ questes/673; DOI : https://doi.org/10.4000/questes.673 


\section{Conclusion : Se prendre au jeu}

\section{Amandine MUSSOU et Laëtitia TABARD}

Jeux d'échecs, de dés, de tables, du cirque, mais aussi «carole», débats de poètes ou fausses confessions, tragédies, olympiades : l'extension du domaine ludique semble infinie. Les différentes contributions de ce bulletin ont rendu compte de l'ampleur du spectre sémantique du jeu médiéval, et à vouloir absolument chercher une unité dans la très grande diversité des pratiques que la notion recouvre, peut-être risque-t-on d'introduire une cohérence factice dans ce qui relève, avant tout, de la fécondité même du ludus. Pourtant, si nous tenterons de donner quelques pistes en ce sens, c'est qu'une impression demeure à la lecture de ces articles : les jeux forment un tout en ce qu'ils sont constitutifs de la société courtoise. L'épisode des jeux du Lancelot en prose prend même tout son sens dans le rapport qu'établit Patrick Moran entre monde courtois, valeurs mondaines et univers du jeu : parce que le «meilleur chevalier du monde » est pris au jeu, il appartient au monde de la cour, et doit s'effacer pour qu'advienne la chevalerie celestielle.

Danser, chanter, jouer aux échecs, interpréter un personnage sont autant de gestes témoignant de l'élégance, du raffinement, de la maîtrise d'un individu qui par le jeu s'amuse de l'ordre du monde et du langage, et domine un code au point de le détourner : il ne se plie pas à la règle mais joue avec elle. Certes, les charmantes dames du Lai d'Ignaure avouent leurs péchés, mais sans honte, et font de la confession, soumission à la loi divine, un moment de vérité paradoxale; et si le jeu des Troyens peut symboliser la cité idéale, organisée mais non tyrannique, c'est aussi parce qu'il incarne un respect de la règle qui ne s'identifie pas à une soumission 
aveugle. Et l'on peut se prendre au jeu et se laisser piéger, tant l'équilibre ludique est fragile. Tel Lancelot tournant en rond sans fin dans le cercle de la carole, le joueur risque d'oublier que cette parenthèse magique doit se refermer, comme cet idéal courtois qui est « rêve d'hérö̈sme et d'amour » ${ }^{1}$.

Cette alliance intime entre aristocratie et jeu, mise en valeur par Daniel Poirion ${ }^{2}$, explique l'évolution du regard sur les jeux, associés à l'oisiveté mais également symboles d'un ordre supérieur. Anne Rochebouet et Anne Salamon étudient dans certains textes « troyens » les variations minimes et pourtant signifiantes qui rendent compte de cette dualité et de cette ambivalence, un auteur mettant l'accent sur l'un ou l'autre de ces aspects. Les échecs, jeu courtois, peuvent être récupérés au profit d'un éloge de la cité idéale incarnée par Troie, tandis que le jeu tel que Simon Gabay l'étudie est considéré comme un trouble de l'ordre par les autorités. Il est alors intégré au fonctionnement social, par la législation. Ces deux articles, que la matière soit fictionnelle ou historique, interrogent le rapport du jeu à la cité : comment insérer dans un ordre social cohérent une pratique gratuite ? Le rapport symbolique entre règle et réglementation confère au jeu la possibilité de devenir un modèle du fonctionnement réglé de la cité et de jouer un rôle social. L'exemple du théâtre montre cependant les limites de cette intégration: les autorités s'efforcent de réguler les pratiques ludiques de façons très diverses, selon des règles qui restent mouvantes.

Si le jeu peut être compris comme un facteur de désordre, sa fécondité, puissance toute positive, a largement été mise en évidence dans

1 Johan Huizinga, L'Automne du Moyen Âge, Paris, Payot, 2002, p. 121 (édition originale : 1919 ; première édition en langue française : Payot, 1932).

${ }^{2}$ Daniel PoIrion, Le Poète et le prince, L'évolution du lyrisme courtois de Guillaume de Machaut à Charles d'Orléans, Paris, PUF, 1965/ Genève, Slatkine Reprints, 1978, p. 73. 
ce bulletin ${ }^{3}$. La contrainte de la règle est en effet souvent créatrice d'un nouvel ordre. Dans les jeux-partis, les auteurs respectent certains codes en se pliant aux attentes formelles et thématiques du genre, afin de se prêter au jeu avec le public de cour. Cependant, Midoriko Kageyama souligne que le respect des normes est parfois un masque dont se parent les poètes pour introduire des éléments nouveaux. Dans le cadre du jeu-parti, les auteurs abordent des thèmes moins attendus, comme celui des cinq sens, et, par ce biais, le débat ludique s'enrichit et s'approfondit jusqu'au niveau de la discussion philosophique, biaisant avec la règle sans y contrevenir. Celle-ci crée un lieu commun, établit le groupe réuni autour d'un principe partagé, par-delà les valeurs discutées. C'est également ce que montre son importance dans l'enseignement, étudiée par Ilse Van der Velden: la codification formelle de la logique et la contrainte des exercices de dispute font de la recherche de la vérité quelque chose qui s'enseigne. Au-delà de cette transmission des règles, les contraintes des exercices scolastiques, une fois formalisée la méthode de discussion, se prêtent aisément à des réécritures ludiques. La règle est transposée dans un contexte différent, où elle est interprétée de manière parodique. L'ordre de la règle est parfois malmené, bouleversé, mais le jeu est réinventé et se trouve de nouvelles bases. Si la règle est contrainte, les contributions de ce bulletin ont beaucoup insisté sur la manière dont les auteurs médiévaux s'en affranchissent et se jouent d'elle. Ainsi le jeu est-il à la fois respect d'une norme et formidable espace de liberté.

\footnotetext{
${ }^{3}$ Sur ce double aspect du jeu, voir l'introduction de Christopher LUCKEN, « Si c'était le Nombre ce serait le Hasard », in Jacques BERChTOLD, Christopher LUCKEN, Stefan SchottKe (dir.), Désordre du jeu - Poétiques ludiques, Genève, Droz, «Recherches et Rencontres » (Publications de la Faculté des Lettres de l'Université de Genève), vol. 6, 1994, p. 9-15.
} 
Activité libre et gratuite selon certains théoriciens ${ }^{4}$, le jeu en dit souvent beaucoup plus qu'il n'y paraît au premier abord sur l'environnement dans lequel il s'insère. En analysant la manière dont les jeux sont intégrés dans la fiction, on s'aperçoit que l'inscription du ludique a souvent une valeur réflexive, métalittéraire. Mathilde Grodet souligne ainsi que l'auteur du Lai d'Ignaure, en mettant en scène le détournement des règles, déjoue les attentes de son lectorat: la fraude du personnage répond à la ruse de l'auteur et c'est une réflexion sur la littérature courtoise et ses codes qui est alors esquissée. Parallèlement, Patrick Moran montre que les jeux périlleux que sont la carole magique et le jeu d'échecs du Lancelot en prose sont à mettre en rapport avec l'architecture générale du roman. Symboles du monde courtois, ils en reproduisent le fonctionnement et les règles, mais en soulignent également les limites et permettent de repenser l'aventure chevaleresque. Inséré à un moment clé du roman, où certains principes commencent à vaciller, cet épisode, aussi insignifiant et léger qu'il paraisse, a une valeur spéculaire et suscite une réflexion sur les modalités d'écriture. L'écriture du jeu signale dans ces deux cas un déplacement, un jeu autour des règles et des stratégies d'écriture. L'inscription d'épisodes ludiques s'accompagne d'une conscience aiguë des principes qui les fondent. Écrire le jeu, c'est donc d'une certaine manière se prendre au jeu de la fiction, et en repenser les règles.

\footnotetext{
${ }^{4}$ Sur cette épineuse question de la gratuité du jeu, voir notamment la synthèse proposée par Michel PICARD dans son introduction «Le jeu et sa fonction », in La Lecture comme jeu. Essai sur la littérature, Paris, Les Éditions de Minuit, «Critique », 1986, p. 13-26 tout particulièrement.
} 